\title{
Grain Size Distribution Obtained from Monte Carlo Simulation and the Analytical Mean Field Model
}

\author{
Chao WANG and Guoquan LIU \\ Materials Science and Engineering School, University of Science and Technology Beijing, Beijing 100083, China. \\ E-mail: wangchaoyu@etang.com, g.liu@ustb.edu.cn
}

(Received on September 12, 2002; accepted in final form on November 27, 2002)

\begin{abstract}
The grain growth process in two dimensions was simulated by Monte Carlo Potts method. The observed quasi stationary state grain size distribution could well be fitted by the Weibull function. It can also be approximated by an analytical distribution with $v$ smaller than 4 , rather than the Hillert distribution $(v=4)$. Our results suggest that here the microstructure would not evolve to the Hillert distribution but to the Weibull function or the analytical distribution with $v<4$. It is also shown that the Weibull function is a better approximation to the quasi stationary state grain size distribution in present work.
\end{abstract}

KEY WORDS: grain growth; grain size distribution; Weibull function; Monte Carlo; Hillert distribution.

\section{Introduction}

Microstructural features determine, to a considerable degree, the properties of materials. Thus control of the microstructure evolution is crucial to the optimization of materials performance through processing. However, it is a challenging problem due to the larger number of variables that must be controlled. Process of grain growth, a technologically important phenomenon, plays an important role in the prediction of microstructure evolution and in practice. So does the modeling of it.

To model the grain growth process, a sound understanding is necessary. Among all the theoretical research of grain growth, Hillert's theory ${ }^{1)}$ has been accepted widely. It predicts that a so-called Hillert grain size distribution (GSD) can be reached from an arbitrary initial microstructure, which has the form:

$$
f(r)=(2 e)^{2} \frac{2 r}{(2-r)^{4}} \exp \left(-\frac{4}{2-r}\right)
$$

where $r$ is the relative grain size. Unfortunately, the Hillert distribution has rarely been observed in practice. ${ }^{2)}$

Brown reexamined the classical Lifshitz and Slyozov ${ }^{3)}$ and Wager," "LSW", theory in the context of particle coarsening and concluded that there are an infinite number of quasi-stationary distributions of which the LSW distributions is an only particular case. ${ }^{5)}$ Brown's view has been clarified and improved in the work of Coughlan and Fortes. ${ }^{6}$ ) Due to Hillert's theory originating from the classical theory of particle coarsening, Haibo $\mathrm{Yu}^{7)}$ and $\mathrm{Rios}^{8)}$ had adapted the work in Ref. 6) to two-dimensional grain growth mutual dependently. They obtained a quasi stationary analytical GSD:

$$
\begin{array}{r}
f(r)=\frac{2 H_{0} r v^{H_{0}}}{\left(r^{2}-v r+v\right)^{1+H_{0}}} \\
\quad \times \exp \left[\frac { - 2 H _ { 0 } v } { \sqrt { 4 v - v ^ { 2 } } } \left(\arctan \left(\frac{2 r-v}{\sqrt{4 v-v^{2}}}\right)\right.\right. \\
\left.\left.\quad+\arctan \left(\frac{v}{\sqrt{4 v-v^{2}}}\right)\right)\right] \ldots \ldots \ldots . . . . . . .
\end{array}
$$

where $v$ is the only parameter, and $H_{0}$ can be numerically determined from:

$$
\int_{0}^{r_{\max }} r f(r) d r=1
$$

where $r_{\max }$ is the maximum of $r$. Equation (2) is valid for $v<4$ and together with Eq. (3) yield a family of quasi stationary GSDs depending on one parameter $v$. As $v$ of Eq. (2) approaches 4, it approaches Eq. (1), the Hillert distribution.

In a recent work, Fayad et al. ${ }^{9)}$ reported that the quasi stationary grain size distribution could be fitted by a Weibull function better than the commonly-cited lognormal or Rayleigh distribution function. The specific form of the Weibull function is:

$$
f(r)=\frac{\beta}{\alpha^{\beta}} r^{\beta-1} \exp \left[-\left(\frac{r}{\alpha}\right)^{\beta}\right]
$$

where $\alpha=1 / \Gamma(1+1 / \beta)$ and $\Gamma$ is the gamma function. This expression only depends on $\beta$.

Equations (2) and (4) are both functions depending on one parameter. They appear quite different in forms. The problem remains: which one could describe the quasi sta- 


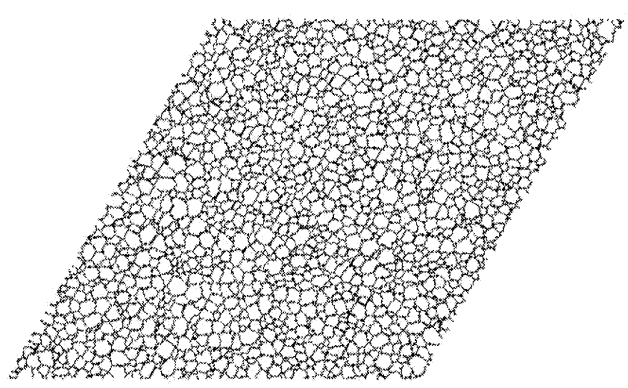

(a)

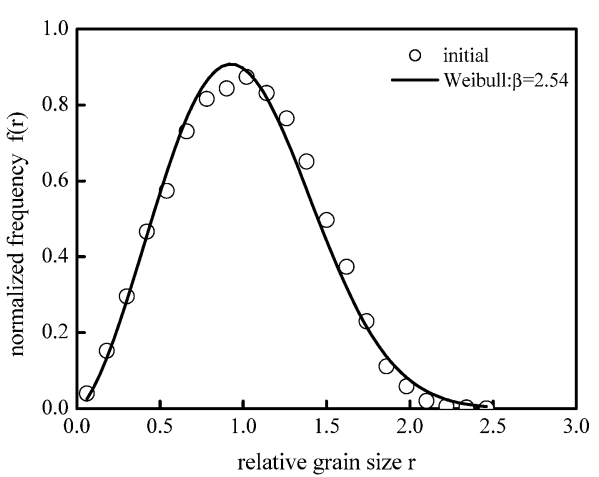

(b)

Fig. 1. (a) The microstructure evolved $200 \mathrm{MCS}$ (the shown is only of $1 / 16$ structure in size of $2000 \times 2000$ sites). (b) Grain size distribution for (a), fit by the Weibull function (solid line).

tionary state GSD better, and is there any possibility that they are internally consistent? This is the interest of this work.

Actually grain growth occurs in three dimensions. However, most of observations are done on two-dimensional cuts to perform studies. And from a theoretical viewpoint, the two-dimensional grain growth is relatively well understood. Therefore, two-dimensional grain growth was simulated to investigate the quasi stationary state grain size distribution.

The Monte Carlo Potts model ${ }^{10-12)}$ is one of the most promising methods to simulate grain growth process and present detail information of microstructure evolution. Here, it was employed to simulate grain growth.

\section{Simulation Procedure}

The microstructure is mapped onto a triangular lattice with full boundary periodic boundary conditions. Each site in the lattice is assigned a positive integer $S_{i}$, corresponding to the orientation of grain which has been mapped onto it. A grain is represented by a collection of lattice sites with a unique orientation number. A grain-boundary segment is defined to lie between sites of unlike orientations. The grain-boundary energy is defined in terms of the lattice site energy:

$$
E_{i}=J \sum_{j=1}^{6}\left(1-\delta s_{i} s_{j}\right)
$$

Where $J$ is a positive constant proportional to grain boundary energy, $S_{i}$ corresponds to the orientation of site $i, \delta s_{i} s_{j}$ is the Kronecker delta function, the summation is taken over all sites $j$, six first nearest-neighbor sites. A grain boundary segment has a positive energy set by $J$, while site in the grain interior have zero energy.

Simulation of grain growth involves the random selection of a site with an orientation $S_{i}$, and its reorientation attempt. The reorientation attempt is restricted to the random site $j$, adjacent to the chosen site. Thus the net energy change associated with reorientation attempt can be expressed:

$$
\Delta E=E_{i 2}-E_{i 1}
$$

where $E_{i 2}$ and $E_{i 1}$ are energy of site $i$ before and after reorientation attempt. The reorientation is accepted only if $\Delta E$ is not larger than zero, which corresponds to a reduction in the local curvature of the grain boundary. Time, in this simulation, is proportional to the number of reorientation number attempts. $N$ reorientations attempts is used as time unit and is referred to as one Monte Carlo step (MCS), where $N$ is the total number of lattice sites.

Simulation was run on a $2000 \times 2000$ triangular lattice starting from an initial amorphous state, i.e., the positive integer from 1 to $2000^{2}$ was assigned to each lattice site.

\section{Results and Discussions}

The microstructure in Fig. 1(a) was obtained after the evolution of $200 \mathrm{MCS}$ and its corresponding GSD was plotted in Fig. 1(b). Least squares fit of the Weibull function was done to the simulated data yielding $\beta=2.54$. Its goodness of fit can be specified by the Chi-square of 0.0014 . This suggests that here the Weibull function is a closer approximation of the GSD.

During the process of grain growth, the time exponent index of the average grain size before $500 \mathrm{MCS}$ is 0.41 . In the later period of grain growth after $500 \mathrm{MCS}$ it is 0.49 (very close to the theoretical value 0.5 ). Figure 2(a) shows the final microstructure of the simulation. In Fig. 2(b), it can be seen that the GSDs basely keep self-similar. The GSDs at time $t=3000,4000,5000 \mathrm{MCS}$ can be well fitted by the Weibull function (the solid line) with $\beta=2.40$ or Eq. (2) (the dash line) with $v=2.81(<4)$. Their fit goodness can be verified by the Chi-square of 0.0055 for the Weibull function, and 0.0077 for Eq. (2). This suggests that the Weibull function approaches the quasi stationary state GSDs more closely than Eq. (2). Meanwhile, the Chisquare value for Eq. (2) is small and the Eq. (2) should be used to describe the quasi stationary state GSDs. However, the imposed Hillert distribution in Fig. 2(b) (the dash dot line) is not suitable to describe the quasi-stationary state GSDs. Or the microstructures would not evolve toward the Hillert distribution. While the quasi-stationary state GSD obtained in the present work can be approximately described by the Weibull function or Eq. (2). This shows that the simulated results from MCP have improved that the quasi-stationary state with $v<4(v=2.81)$ should exist. It 


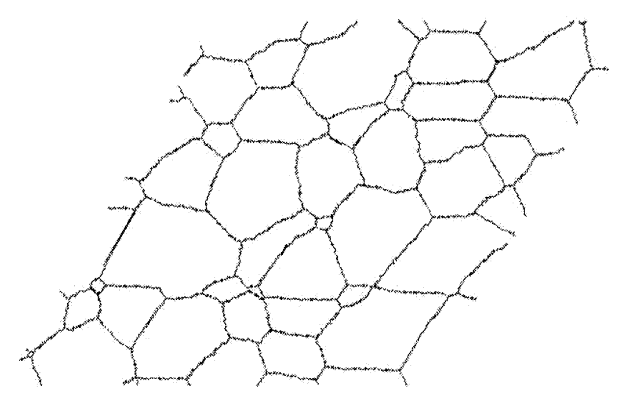

(a)

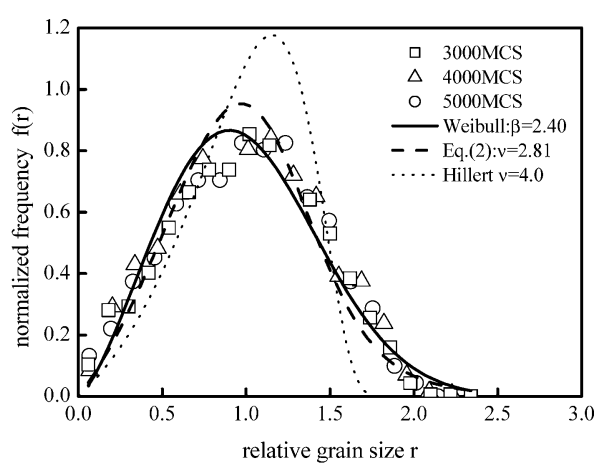

(b)

Fig. 2. (a) The microstructure at $5000 \mathrm{MCS}$ (the shown is only of $1 / 16$ structure in size of $2000 \times 2000$ sites). (b) The GSDs at 3000,4000 , and $5000 \mathrm{MCS}$, compared with the Weibull function (solid line), Eq. (2) (dash line), and the Hillert distribution (dash dot line).

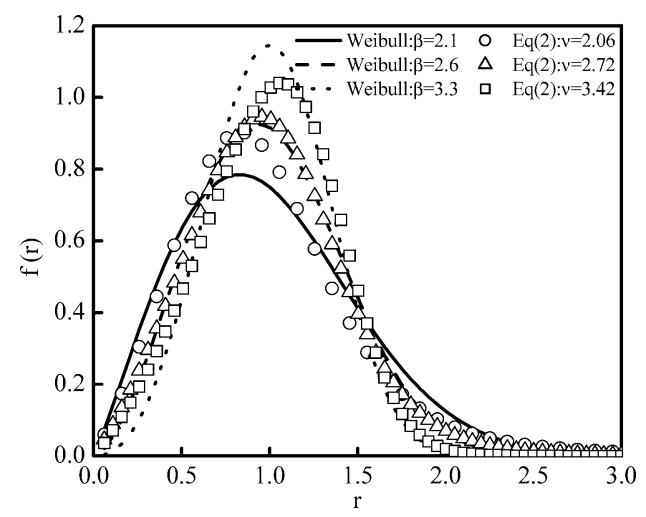

Fig. 3. Comparisons between the Weibull function and the Eq. (2).

also suggests that there is compatibility between the Monte Carlo Potts method and the mean field model.

Both the Weibull function and the Eq. (2) are a family of distributions depending on $\beta$ or $v$. Equation (2) has a complex form derived from the rigorous theoretical deduction. The form of the Weibull function is simple. However, comparisons of the two functions show that: as $\beta$ of the Weibull function is in the range of 2.1-3.3, and $v$ of Eq. (2) in the range of 2.06-3.42, the two functions almost have the same value, thus they can be alternated each other, as shown in Fig. 3. Then it is possible that in such a range Eq. (2) may be approximately expressed by the Weibull function. It is self-evident that the quasi-stationary GSD in Fig. 2 can be well described by the two functions. Out of the range, the alteration of the two functions would produce a lager error. But it is unknown whether both of them can describe the quasi-stationary GSD or not since such a GSD has not been observed in present work.

\section{Conclusions}

Monte Carlo Potts simulation was performed starting from an initial amorphous microstructure. The obtained quasi stationary state grain size distribution deviates from the Hillert distribution. However, it can be fitted by the Weibull function and an analytical distribution (with $v<4$ ) obtained from the theoretical deduction as well. And the Weibull function is a better approximation to it. This shows that the microstructures would not evolve to the Hillert distribution but to the Weibull function or the complicated analytical distribution with $v<4$. It proves that the non-Hillert $(v<4)$ quasi stationary state grain size distribution would exist. In a certain range of parameter, the Weibull function may be an alternative form of the complicated analytical distribution.

\section{Acknowledgements}

The authors are grateful for financial supports from the National Natural Science Foundation of China through contact No. 50171008.

\section{REFERENCES}

1) M. Hillert: Acta Metall., 13 (1965), No. 3, 227.

2) H. V. Atkinson: Acta Metall., 36 (1988), No. 3, 469.

3) I. M. Lifshitz and V. V. Slyozov: J. Phys. Chem. Solids USSR, 19 (1961), 315.

4) C. Wagner: Z. Elektrochem., 65 (1961), 581.

5) L. C. Brown: Acta Metall., 37 (1989), No. 1, 71.

6) S. D. Coughlan and M. A. Fortes: Scr. Metall. Mater., 28 (1993), 1471.

7) H. Yu: Doctorate dissertation, University of Science and Technology Beijing, Beijing, (1997), 5.

8) P. R. Rios: Scr. Mater, 40 (1999), No. 6, 665.

9) W. Fayad, C. V. Thompson and H. J. Frost: Scr. Mater., 40 (1999), No. 10, 1199.

10) B. Radhakrishnan and T. Zacharia: Metall. Mater. Trans., 26A (1995), No. 1, 167

11) Y. Saito: ISIJ Int., 38 (1998), No. 6, 559.

12) X. Song and G. .Liu: J. Mater. Sci., 34 (1999), 2433. 